\title{
Efeito da remoção seletiva da sutura no astigmatismo nos transplantes de córnea pós-ceratocone
}

\section{The effect of selective suture removal on astigmatism following penetrating keratoplasty in keratoconus}

Fernando Moro', Érica Galúcio², Ediberto de Magalhães², Karine Borges Marques Moysés', Victor Coronado Antunes', Tadeu Cvintal ${ }^{3}$

\section{RESUMO}

Objetivo: Demonstrar o efeito e os resultados da remoção seletiva da sutura sobre o astigmatismo nos três primeiros meses de transplante de córnea pós-ceratocone. Métodos: Realizamos um estudo longitudinal observacional retrospectivo, revisando aleatoriamente os prontuários médicos de 50 pacientes, submetidos à transplante penetrante de córnea por ceratocone, realizados pelo mesmo cirurgião, no período entre $2000 \mathrm{e}$ 2002, no Instituto de Oftalmologia Tadeu Cvintal. Os transplantes foram realizados com a mesma técnica cirúrgica com oito pontos isolados e sutura contínua e controle ceratoscópico per-operatório. Em todas as visitas foram mensuradas a melhor acuidade visual corrigida, ceratometria e refração. A remoção seletiva da sutura e redistribuição da sutura contínua ocorreu invariavelmente no primeiro mês de pós-operatório. Resultados: No primeiro mês de pós-operatório, antes do início de remoção de suturas a acuidade visual média pré-remoção foi de $0,4 \log$ MAR. Após remoção seletiva escalonada de suturas e estabilização do astigmatismo, a acuidade visual média pósremoção foi de $0,07 \log$ MAR, diferença estatisticamente significativa $(\mathrm{p}<0,0001)$. A ceratometria média pré-operatória foi de 2,8D, 3,8D com 30 dias de pós-operatório e de 2,8D pós-remoção de suturas e estabilização do astigmatismo com 90 dias de pósoperatório, diferenças estatisticamente significante $(\mathrm{p}=0,0229)$. A redistribuição da sutura contínua foi realizada em $30 \%$ dos pacientes. Obteve-se melhora da acuidade visual em 100\% dos pacientes. Conclusão: $\mathrm{O}$ transplante penetrante de córnea em pacientes portadores de ceratocone constitui uma excelente alternativa para recuperação da acuidade visual. A remoção seletiva de pontos isolados e redistribuição da sutura contínua no primeiro mês de pós-operatório promoveram melhora significativa da visão com recuperação visual precoce.

Descritores:Transplante de córnea; Ceratoplastia penetrante; Astigmatismo/prevenção \& controle; Ceratocone; Suturas; Doenças da córnea; Estudos retrospectivos

\footnotetext{
${ }^{1}$ Assistentes do departamento de córnea do Instituto de Oftalmologia Tadeu Cvintal - São Paulo (SP), Brasil;

${ }^{2}$ Residentes do Instituto de Oftalmologia Tadeu Cvintal - São Paulo (SP), Brasil;

${ }^{3}$ Diretor do Instituto de Oftalmologia Tadeu Cvintal - São Paulo (SP), Brasil.
}

Recebido para publicação em: 09/07/2007 - Aceito para publicação em 26/10/2007 


\section{INTRODUÇÃO}

$\mathbf{O}$ transplante penetrante de córnea leva a uma melhora significativa da acuidade visual em pacientes com ceratocone tanto por diminuir a curvatura excessiva da córnea como por corrigir o astigmatismo. Para isso, contribuíram de forma significativa os avanços com relação a instrumental cirúrgico, técnicas de trepanação, técnicas e manejo na remoção das suturas. Atualmente, o êxito de uma ceratoplastia é avaliado em termos de resultados refrativos, uma vez que a transparência do enxerto é obtida, praticamente, em todos os casos. No ceratocone, quando a cirurgia se torna necessária do ponto de vista anatômico e funcional, a córnea quase sempre se encontra com protrusão acentuada o que impossibilita a avaliação refratométrica adequada e até mesmo adaptação de lentes de contato. $\mathrm{O}$ transplante penetrante de córnea e o adequado manejo pós-operatório da sutura possibilitam recuperação visual significativa nestes pacientes que na maior parte são pacientes jovens e em idade francamente produtiva ${ }^{(1)}$.

\section{Métodos}

Realizamos um estudo longitudinal observacional por meio de revisão aleatória e retrospectiva de 50 casos de transplante penetrante de córnea por ceratocone, realizados pelo mesmo cirurgião, no período entre 2000 e 2002.

Os transplantes foram realizados com a mesma técnica cirúrgica com oito pontos isolados e sutura contínua utilizando o fio mononáilon 10.0 e controle ceratoscópico per-operatório. $\mathrm{O}$ diâmetro doador mais utilizado foi $8,5 \mathrm{~mm}$ e receptor $8,0 \mathrm{~mm}$. As córneas doadoras foram preservadas em meio Optisol. Todas as cirurgias foram realizadas com anestesia geral e seguiram o mesmo protocolo pós-operatório. As visitas ocorreram no período pré-operatório e primeiro, segundo e terceiro dia de pós-operatório para troca do curativo oclusivo, uma semana, trinta, sessenta e noventa dias de pós-operatório. Em todas as visitas foram anotadas a melhor acuidade visual corrigida, ceratometria e refração.

Os parâmetros utilizados para avaliação do astigmatismo foram a ceratometria com o ceratômetro de Bausch \& Lomb, ceratoscopia computadorizada e a refração dinâmica.

Utilizamos o ponto contínuo associado a oito pontos isolados em todos os casos estudados.

A remoção seletiva e redistribuição dos pontos ocorreram invariavelmente no primeiro mês, removendo os pontos isolados seletivamente. Se não obtivésse-

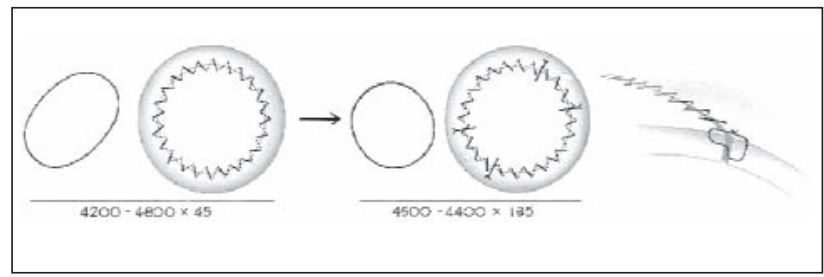

Figura 1: Remoção do ponto isolado orientado pela ceratoscopia

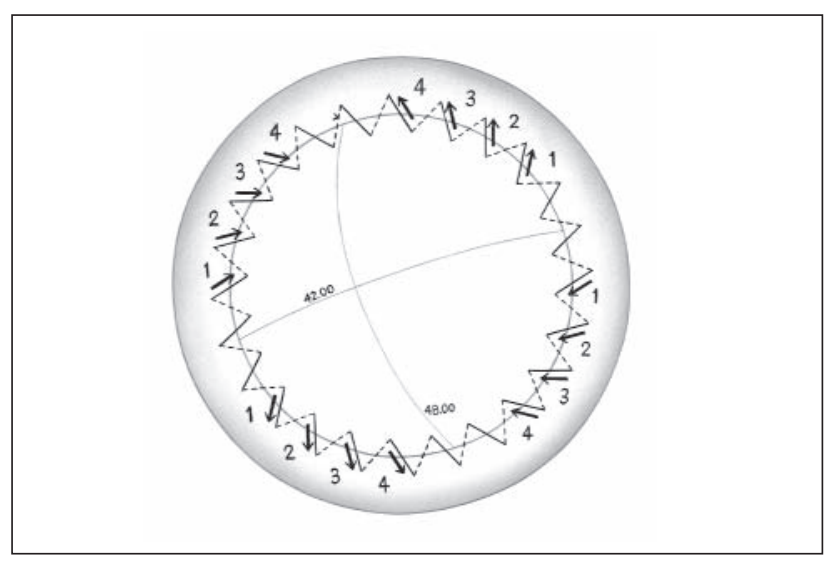

Figura 2: Redistribuição da sutura contínua ${ }^{5}$

mos o resultado procurado, em seguida a redistribuição do contínuo era realizada.

A redistribuição da sutura contínua foi indicada na primeira semana nos astigmatismos maiores de três dioptrias, quando não obtivemos bons resultados com a remoção seletiva da sutura isolada (Figura 2).

Uma vez que o hemimeridiano mais curvo foi corretamente determinado, identificamos na lâmpada de fenda a sutura mais apertada naquele local e sob anestesia tópica a sutura era seccionada e removida.

Se a ceratometria ou a videoceratoscopia computadorizada revelasse presença de astigmatismo regular e simétrico, as suturas eram removidas simetricamente, em número de uma ou duas suturas em cada hemimeridiano, dependendo do grau do astigmatismo (astigmatismos maiores que três dioptrias). Nos casos de irregularidade, as suturas removidas obedeciam ao eixo do hemimeridiano mais curvo.

Se o astigmatismo presente fosse assimétrico, as suturas também eram removidas assimetricamente, devendo ser em maior número no hemimeridiano mais curvo. Imediatamente, após a remoção da sutura, as alterações no astigmatismo ceratométrico e refracional eram observadas.

Uma gota de colírio antibiótico (Dexafenicol ${ }^{\circledR}$ ), sem conservante, associado à dexametasona a $0,1 \%$, era instilada quatro vezes ao dia, além de lubrificação intensa com colírio (Freshtears ${ }^{\complement}$ ) foram prescritos no pri- 


\section{Gráfico 1}

Média da melhor acuidade visual logMAR corrigida no pré-operatório e após 30 e 90 dias da realização do transplante penetrante de córnea

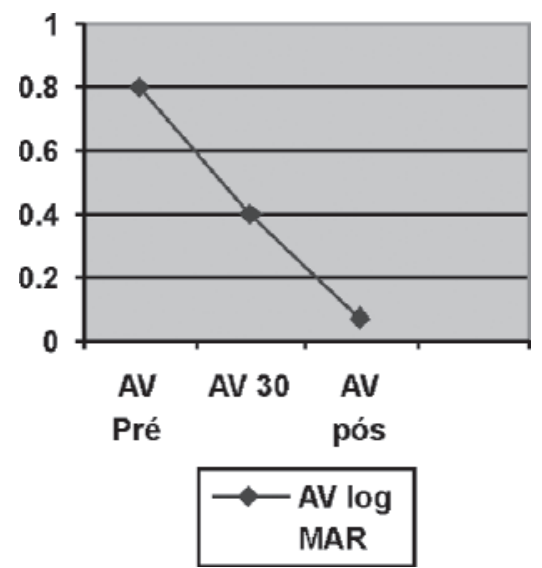

\section{Gráfico 3}

Porcentagem de miopia e hipermetropia com relação à refração esférica final

Refração esférica final

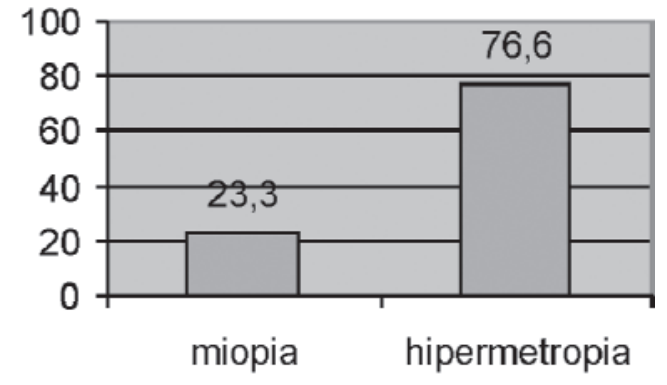

$\square$ Refração esférica final meiro mês de pós-operatório.

Caso após toda sutura ser removida ainda persistisse astigmatismo elevado, uma ressutura era realizada.

Os resultados de variáveis contínuas foram expressos por suas médias aritméticas e respectivos desvios-padrão, ou pelos valores de medianas e respectivas distâncias interquartil. Foi utilizado o teste t-Student para amostras pareadas na avaliação de possíveis diferenças entre médias. $\mathrm{O}$ teste de Wilcoxon foi usado para verificar possíveis diferenças entre medianas; $p<0,05$ foi utilizado para rejeitar a hipótese de nulidade.
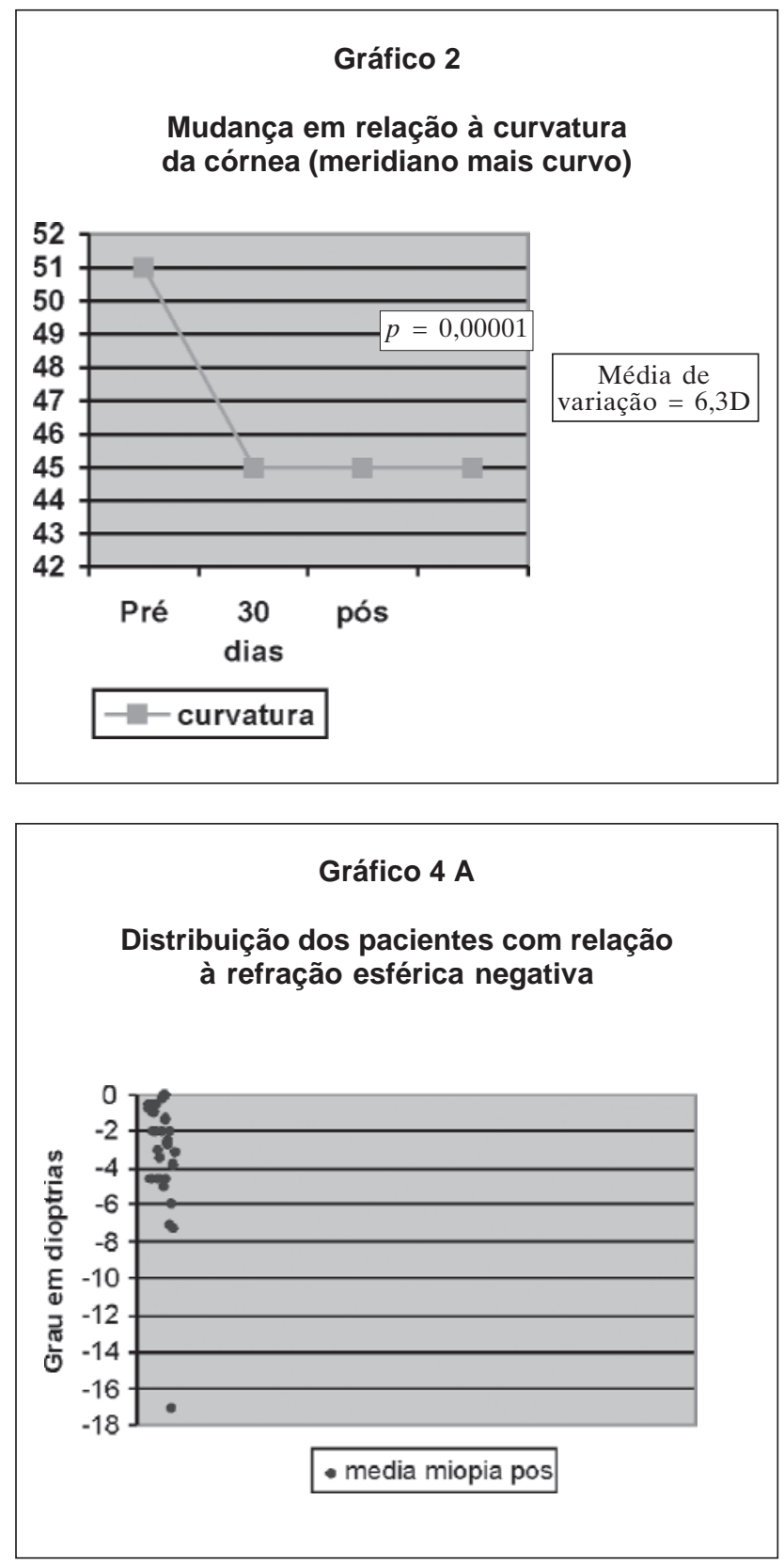

Tabela 1

Valores do astigmatismo topográfico e refracional

\begin{tabular}{lcc}
\hline & \multicolumn{2}{c}{ Astigmatismo } \\
& Topográfico & Refracional \\
\hline Pré & 2,8 & \\
30 d & 3,8 & 3,88 \\
Pós & 2,8 & 2.54 \\
RA & 0 & 1.04 \\
$\boldsymbol{p}$ & 0,0229 & 0,0001 \\
\hline
\end{tabular}




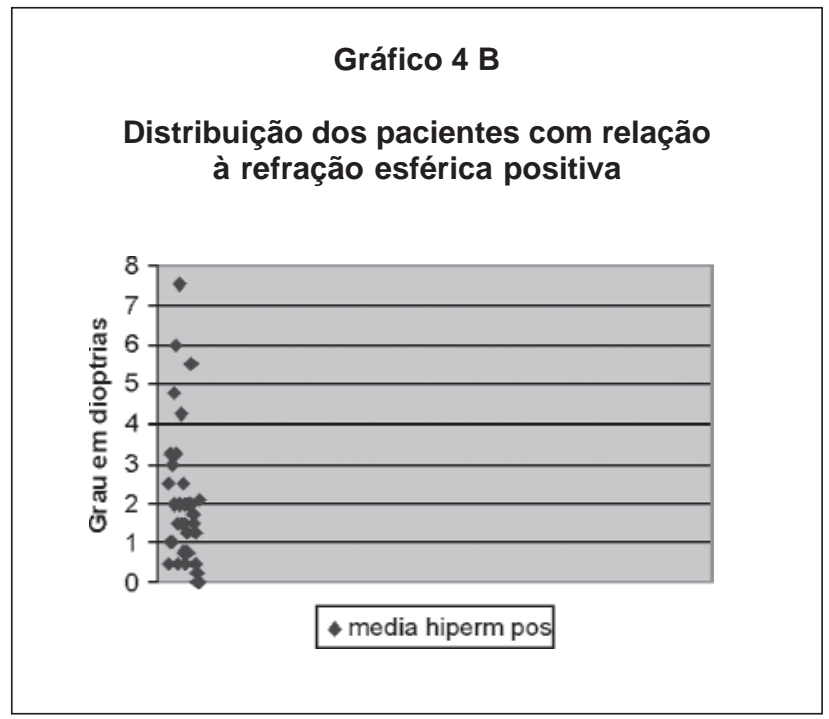

\section{Resultados}

A média de idade dos pacientes foi de 24,8 anos, variando de 11 a 50 anos. A melhor acuidade visual corrigida pré-operatória média foi $0,801 \log$ MAR. No primeiro mês de pós-operatório, antes do início de remoção de suturas, a acuidade visual média pré-remoção foi 0,405 $\log$ MAR.Após remoção seletiva escalonada de suturas e estabilização do astigmatismo, a acuidade visual corrigida média pós-remoção foi $0,073 \log \mathrm{MAR}$, com um valor estatisticamente significativo $(\mathrm{p}<0,0001)$ (Gráfico 1$)$.

$\mathrm{O}$ astigmatismo pré-operatório ceratométrico ou topográfico, antes da remoção de suturas e após remoção de suturas, com estabilização do astigmatismo em médio prazo, variaram da seguinte forma: a ceratometria média pré-operatória era de 2,8D; $3,8 \mathrm{D}$ no período de 30 dias de pós-operatório e antes da remoção da sutura e média de 2,8D após a remoção de suturas e estabilização do astigmatismo 90 dias de pós-operatório, diferenças estatisticamente significantes $(\mathrm{p}=0,0229)$. Vale a pena lembrar que o astigmatismo pré-operatório corresponde a ceratometria média central de $3 \mathrm{~mm}$ e a maior parte dos ceratocones apresentam o ápice do cone inferior, além do fato do astigmatismo na maioria das vezes ser assimétrico no pré-operatório (Tabela 1).

Comparando-se, entretanto, a mudança na curvatura da córnea, no meridiano mais curvo, acharemos valores estatisticamente significantes $(\mathrm{p}=0,0000001)$ que variaram de 51,5 (ceratometria média pré-operatória) a 45,2 (ceratometria média pós-operatória) (Gráfico 2).

Com relação a refração esférica final, 23,3\% dos pacientes apresentaram miopia que variou de $-0,75 \mathrm{D}$ a -15,00D com uma média de -3.17D. Aproximadamente,

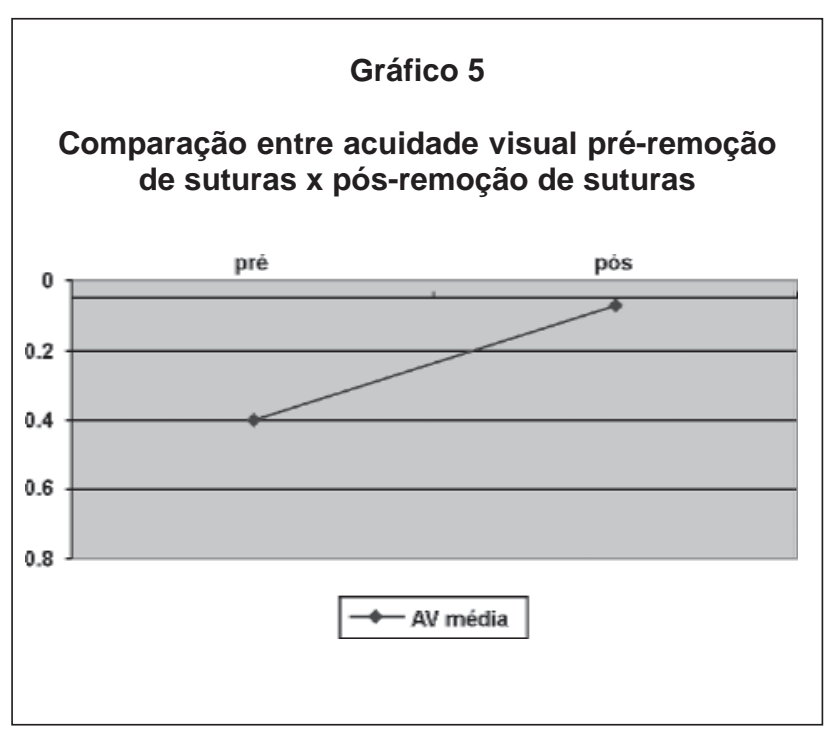

$76,6 \%$ dos pacientes apresentaram baixos graus de hipermetropia, variando entre $+0.25 \mathrm{a}+7,00 \mathrm{D}$ com uma média de +2,07D, e emetropia (Gráficos 3 e 4 A e B).

Finalmente, a melhor acuidade visual corrigida melhorou em $100 \%$ dos pacientes (Gráfico 5). A redistribuição da sutura contínua foi realizada em 30\% dos pacientes.

\section{Discussão}

$\mathrm{O}$ astigmatismo constitui uma das mais freqüentes causas de baixa acuidade visual no pós-operatório do transplante de córnea, tanto a curto como em longo prazo $^{(2)}$. Astigmatismo excessivo, astigmatismo irregular ou assimétrico são uma das principais causas de impossibilidade de correção óptica com óculos, podendo ainda condicionar anormalidades do filme lacrimal e dificuldades na adaptação de lentes de contato $^{(3)}$.

A ametropia esférica e astigmatismo elevado, ainda são, na atualidade, a principal causa de insucesso funcional no transplante penetrante de córnea ${ }^{(4)}$. Observou-se que mesmo pós-transplante, uma taxa elevada de pacientes apresentam graus de astigmatismo incompatíveis com uma boa acuidade visual ${ }^{(5)}$. Na maioria dos casos, este problema se deve a falha no manejo adequado com relação às suturas corneanas durante o pós-operatório, já que estudos demonstraram que tanto o grau, quanto a técnica de sutura não influenciam a acuidade visual final ${ }^{(5)}$.

Existem muitos mecanismos para induzir o astigmatismo num transplante de córnea como: trepanação oval ou oblíqua do doador ou receptor, descentração da trepanação do doador ou receptor, restos de Descemet do tecido doador ou receptor, disparidade 
de espessura entre o doador e o receptor, doenças na periferia da córnea receptora, vascularização da periferia da córnea receptora, cicatrização irregular, irregularidade ou desequilíbrio da tensão das suturas, etc ${ }^{(6)}$.

Soma-se o fato do ceratocone ser afecção potencialmente condicionadora de astigmatismo no pós-operatório, uma vez que a córnea a ser trepanada apresenta importante deformação periférica. Além disso, áreas distróficas localizadas que provocam afrouxamento prematuro dos pontos correspondentes com conseqüente indução de protrusão localizada e astigmatismo assimétrico $^{(5)}$.

A presença de leucomas cicatriciais que se estendem até a periferia, com retração do tecido, provocam uma deformidade do contorno da trepanação, induzindo o astigmatismo pós-operatório. A presença de vascularização na periferia em pacientes usuários crônicos, às vezes desconfortavelmente adaptados às lentes de contato, inicialmente provoca edema, afrouxando os pontos e causando abaulamento. Posteriormente, esta área vascularizada apresenta uma maior migração de fibroblastos e com isso uma fibrose mais intensa, determinando desequilíbrio na cicatrização e indução a $\operatorname{astigmatismo~tardio~}^{(7)}$.

O conhecimento detalhado destes mecanismos orienta o cirurgião para melhor utilizar os recursos de técnica cirúrgica para anular os diferentes mecanismos, à medida que são detectados.

Ajustes intra-operatórios e pós-operatórios são preconizados para se obter rápida reabilitação visual e estabilidade refracional ${ }^{(8)}$.

A resposta da superfície corneana frente à remoção da sutura é imprevisível e complexa. O semimeridiano mais curvo na zona dos $3 \mathrm{~mm}$ centrais mostrou máxima alteração uma hora após a remoção da sutura ${ }^{(9)}$.

Apesar disso, a remoção gradual e seletiva de suturas isoladas é o método mais simples e eficaz para reduzir o astigmatismo no pós operatório de transplante de córnea, proporcionando rápida reabilitação visual ${ }^{(10-14)}$.

Quando existe combinação de sutura isolada e contínua, a redistribuição da sutura contínua é também eficaz no arredondamento da imagem ceratoscópica final ${ }^{(8)}$.

Com o objetivo de diminuir o astigmatismo e melhorar a acuidade visual o mais rápido possível, a redistribuição da sutura contínua na lâmpada de fenda pós-transplante de córnea é o método mais simples.

A redistribuição da sutura contínua, quando realizada precocemente no primeiro dia de pós-operatório, obtém resultados previsíveis, estáveis e de realização mais fácil ${ }^{(3)}$.

Realizamos a redistribuição na primeira se- mana de pós-operatório, pois a partir da segunda semana a manobra de redistribuição é difícil e em geral ineficaz ${ }^{(2)}$.

A eficiência do método depende da correta identificação da sutura indutora do astigmatismo, através da avaliação do hemimeridiano mais curvo e da sutura correspondente.

O ceratômetro Bausch \& Lomb fornece informações quantitativas e qualitativas do astigmatismo, uma vez que a porção mais achatada da imagem ceratoscópica corresponde ao meridiano mais curvo da córnea, e a mais ovalada ao meridiano mais plano. É possível utilizar estas imagens para orientação na remoção ou redistribuição das suturas.

A sutura contínua no transplante de córnea apresenta algumas vantagens tais como, mais rápida de ser realizada do que a sutura separada, e determina menor reação inflamatória, porém não pode ser removida enquanto não houver completa cicatrização.

Por isso quando um astigmatismo acentuado se estabelece no pós-operatório o seu controle é mais difícil que nos pontos isolados ${ }^{(2)}$.

No entanto, transplantes com sutura contínua apresentam melhores resultados em curto prazo e menor astigmatismo, quando comparados aos transplantes com sutura isolada. A sutura que menos induziu o astigmatismo foi a de 8 pontos separados e um contínuo, proporcionando maior estabilidade no astigmatismo, garantindo o melhor resultado final, inclusive em relação à acuidade visual ${ }^{(15)}$.

O astigmatismo é comparável independentemente da técnica de sutura em pacientes com ceratocone, quando o acompanhamento pós-operatório e ajuste e ou remoção seletiva da sutura é realizada de forma adequada ${ }^{(16)}$.

Quando utilizamos o ponto contínuo associado a oito pontos isolados, o astigmatismo é medido com segurança, primeiramente com a remoção dos pontos isolados seletivamente, o que é feito no período de uma a quatro semanas de pós-operatório. Se não obtemos o resultado procurado, tentamos em seguida a redistribuição do contínuo ${ }^{(5)}$.

\section{Conclusão}

O transplante penetrante de córnea em pacientes portadores de ceratocone constitui uma excelente alternativa para melhora da acuidade visual e qualidade de vida. A remoção seletiva de pontos isolados e redistribuição da sutura contínua no primeiro mês de pós-operatório promovem resultados visuais excelentes e recuperação visual precoce. 


\begin{abstract}
Purpose: To analyse the corneal astigmatism after transplantation as well as to demonstrate how postsurgical handling of sutures affects the refractive and $v i$ sual outcome in patients submitted to cornea transplantation by keratoconus with three to six years of follow-up. Methods: Non-controlled longitudinal observational secondary clinical study. Fifty records of patients submitted to penetrating keratoplasty between 2000 and 2002 have been reviewed, including 50 eyes of 50 patients operated under the same surgical technique by the same surgeon. Follow-up time ranging from three to six years. Main outcome measures were keratometry, static refraction and computadorized keratometry. Tstudent and Wilcoxon tests were used for paired samples to assess respectively possible differences between means and medians. Results: The average patient age was 24.8 years. The average of the pre-visual sharpness was $0.8 \mathrm{log}$ $M A R$ and changed to $0.4 \log M A R$ before removing the sutures and to $0.4 \log$ MAR after the handling/removal of sutures $(p<0.0001)$. The topographic astigmatism varied from $2.8 D$ before transplantation to $3.8 D$ in the following 30 days and reached an average of 2.8 after the handling/ removal of sutures $(p=0.0229)$. The corneal curvature changed from 51.5 to $45.2(p=0.0000001) .23 .3 \%$ of the patients presented myopic and hypermetropic deviation averages of respectively $-3.17 D$ and $+2.07 D .113$ days was the average for the beginning of the suture removal. Conclusion: Although the selective and stepwise removal of sutures requires more visits of the patient to the doctor in the first months that follow the penetrating keratoplasty, the good visual and refractive results corroborate not only the importance of the post-surgical attendance, but also the identification and immediate intervention in the selective suture removal of keratoplasty.
\end{abstract}

Keywords: Corneal transplantation; keratoplasty, penetrating; Astigmatism/prevention \& control; Sutures; keratoconus; Retrospective studies

\section{REFERÊNCIAS}

1. Szentmáry N, Seitz B, Langenbucher A, Naumann GO. Repeat keratoplasty for correction of high or irregular postkeratoplasty astigmatism in clear corneal grafts. Am J Ophthalmol. 2005; 139(5):826-30.

2. Tuft SJ, Gregory W. Long-term refraction and keratometry after penetrating keratoplasty for keratoconus. Cornea. 1995; 14(6):614-7.

3. McNeill, Wessels IF. Adjustment of single continuous suture to control astigmatism after penetrating keratoplasty. Refract Corneal Surg. 1989; 5(4): 216-23.
4. Judge D, Gordon L, Vander Zwaag R, Wood TO. Refractive versus keratometric astigmatism postkeratoplasty. Refract Corneal Surg. 1990; 6(3):174-8. Comment in: Refract Corneal Surg. 1990; 6(6):472-3.

5. Munarin MA, Cvintal T. Astigmatismo na presença de suturas. In: Cvintal T. Complicações do transplante de córnea. São Paulo: Livraria Santos; 2004. p.263-71.

6. de Toledo JA, de la Paz MF, Barraquer RI, Barraquer J. Longterm progression of astigmatism after penetrating keratoplasty for keratoconus: evidence of late recurrence. Cornea. 2003; 22(4):317-23.

7. Langenbucher A, Seitz B. Changes in corneal power and refraction due to sequential suture removal following nonmechanical penetrating keratoplasty in eyes with keratoconus. Am J Ophthalmol. 2006; 141(2):287-93.

8. Serdarevic ON, Renard GJ, Pouliquen Y. Randomized clinical trial of penetrating keratoplasty. Before and after suture removal comparison of intraoperative and postoperative suture adjustment. Ophthalmology. 1995; 102(10):1497-503.

9. Solomon A, Siganos CS, Frucht-Pery J. Corneal dynamics after single interrupted suture removal following penetrating keratoplasty. J Refract Surg. 1999; 15(4):475-80.

10. Feldman ST, Brown SI. Reduction of astigmatism after keratoplasty. Am J Ophthalmol. 1987; 103(3 Pt 2):477-8.

11. Mader TH, Yuan R, Lynn MJ, Stulting RD, Wilson LA, Waring GO 3rd. Changes in keratometric astigmatism after suture removal more than one year after penetrating keratoplasty. Ophthalmology. 1993; 100(1):119-26; discussion 127. Comment in: Ophthalmology. 1993; 100(6):797.

12. Burk LL, Waring GO 3rd, Harris DJ Jr. Simultaneous and sequential selective suture removal to reduce astigmatism after penetrating keratoplasty. Refract Corneal Surg. 1990; 6(3):179-87.

13. Binder PS. Selective suture removal can reduce postkeratoplasty astigmatism. Ophthalmology. 1985; 92(10):1412-6.

14. Binder PS. The effect of suture removal on postkeratoplasty astigmatism. Am J Ophthalmol. 1988; 105(6):637-45.

15. Lambert LC, Hamada A, Munarin MAM, Cvintal T. A importância da sutura com 24 pontos no transplante de córnea. Rev Bras Oftalmol. 1994; 53(6):17-20.

16. Javadi MA, Naderi M, Zare M, Jenaban A, Rabei HM, Anissian A. Comparison of the effect of three suturing techniques on postkeratoplasty astigmatism in keratoconus. Cornea. 2006; 25(9):1029-33.

\section{ENDEREÇO PARA CORRESPONDÊNCIA:}

Fernando Moro

Rua Saul Navarro, 240 - Praia do Canto

CEP 29057-240 - Vitória - Espírito Santo

fmoro@uol.com.br 